\title{
Update on use of chloroquine/hydroxychloroquine to treat coronavirus disease 2019 (COVID-19)
}

\author{
Jianjun Gao ${ }^{1, *}$, Shasha $\mathrm{Hu}^{2}$ \\ ${ }^{1}$ Department of Pharmacology, School of Pharmacy, Qingdao University, Qingdao, China; \\ ${ }^{2}$ Department of Pathology, the Affiliated Hospital of Qingdao University, Qingdao, China.
}

\begin{abstract}
SUMMARY Drugs that are specifically efficacious against SARS-CoV-2 have yet to be established. Chloroquine and hydroxychloroquine have garnered considerable attention for their potential to treat coronavirus disease 2019 (COVID-19). Increasing evidence obtained from completed clinical studies indicates the prospects for chloroquine/hydroxychloroquine to treat COVID-19. More randomized control clinical studies are warranted to determine the feasibility of these two drugs in treating COVID-19.
\end{abstract}

Keywords SARS-CoV-2, COVID-19, chloroquine, hydroxychloroquine

Coronavirus disease 2019 (COVID-19) has caused more than 1 million confirmed cases and approximately 70,000 deaths worldwide as of April 6, 2020 (1). Drugs that are specifically efficacious against SARS-CoV-2 have yet to be established. Chloroquine and hydroxychloroquine have garnered considerable attention for their potential to treat COVID-19. In China, chloroquine was added to the "Guidance for Corona Virus Disease 2019: Prevention, Control, Diagnosis, and Management" issued by the National Health Commission on February 18, 2020 (2). The U.S. Food and Drug Administration (FDA) issued an emergency use authorization for chloroquine/ hydroxychloroquine to treat COVID-19 on March 28, 2020 (3). The European Medicines Agency contended that the two drugs should be used in clinical trials or national emergency use programs for the treatment of COVID-19 on April 1, 2020 (4). The low cost and easy availability of chloroquine/hydroxychloroquine may help to curb this global public health emergency if their efficacy and safety are ultimately verified in clinical studies.

Chloroquine phosphate is the first drug reported to display efficacy against COVID-19 in early clinical studies in China $(5,6)$. Based on this encouraging finding, chloroquine phosphate was added to the Sixth Edition of the Guidance for tentative treatment of COVID-19 (2). The Sixth Edition recommended that adult patients in whom chloroquine is not contraindicated should take chloroquine phosphate tablets, $500 \mathrm{mg}$ (300 mg for chloroquine) twice a day for no more than 10 days (7). To reduce the risk of adverse effects of chloroquine, the Seventh Edition of the Guidance, issued on March
3,2020 , recommends a reduced dosage and shortened duration of treatment (8). Specifically, the dosage of chloroquine phosphate for adult patients (ages 18-65) with a body weight greater than $50 \mathrm{~kg}$ is $500 \mathrm{mg}$ twice a day for 7 days and that for adult patients (ages 18-65) with a body weight less than $50 \mathrm{~kg}$ is $500 \mathrm{mg}$ twice a day for the first 2 days and $500 \mathrm{mg}$ once a day for the following 3-7 days (8). Regarding the treatment strategy, chloroquine and another antiviral favipiravir that also displayed efficacy in clinical trials are highlighted for their use in mild and moderate COVID-19 cases to prevent disease progression (9).

In a pilot study (10) aiming to evaluate the efficacy and safety of chloroquine in inpatients with COVID-19, 10 patients ( 3 with severe disease and 7 with moderate disease) received chloroquine phosphate $500 \mathrm{mg}$ orally twice a day for 10 days, and 12 patients (5 with severe disease and 7 with moderate disease) received lopinavir/ritonavir $400 / 100 \mathrm{mg}$ orally twice a day for 10 days. Chloroquine was slightly superior to lopinavir/ ritonavir in terms of virus-negative conversion since the chloroquine group tested negative for SARS-CoV-2 at a slightly higher rate than the control group on day 7, day 10, and day 14 post-treatment (10). However, chloroquine was superior to lopinavir/ritonavir in improving the radiological appearance of the lungs and decreasing the duration of hospitalization. Adverse events including vomiting, abdominal pain, nausea, diarrhea, a rash or itch, a cough, and shortness of breath were observed in the chloroquine group, but chloroquine was not discontinued in any of the patients during the treatment period. The efficacy and safety of chloroquine 
phosphate was also tested in an open-label, multicenter, non-randomized trial in China and the results were disclosed in an international meeting to share experiences to prevent and control COVID-19 (11). Chloroquine demonstrated significant efficacy in reducing the time until virus-negative conversion and returning body temperature to normal (11).

Hydroxychloroquine, a more tolerable derivative of chloroquine, also displayed potent activity against SARS-CoV-2 in vitro (12). A study in France by Raoult et al. (13) evaluated the efficacy of hydroxychloroquine in 26 patients with COVID-19 and found that administration of hydroxychloroquine $200 \mathrm{mg}$, three times a day led to a significant reduction in viral carriage on day 6 post-treatment compared to that in the control group, which did not receive hydroxychloroquine. That study also revealed that adding azithromycin to hydroxychloroquine appears to more efficiently clear the virus. A subsequent study by Raoult et al. (14) involving 80 patients with COVID-19 found that treatment with hydroxychloroquine and azithromycin resulted in a rapid decline in the nasopharyngeal viral load, with a virusnegative rate of $83 \%$ on day 7 and a rate of $93 \%$ on day 8 . Although a control group was not included in that study, its results suggest the potential of hydroxychloroquine to treat COVID-19 given that the recorded median duration of viral shedding is 20 days in survivors (15). In addition to the studies cited above, a Chinese team recently released the results of a clinical study on hydroxychloroquine to treat COVID-19 (16). That study divided 62 patients into a hydroxychloroquine group and a control group at a ratio of $1: 1$. Patients in the control group received standard treatment including oxygen therapy, antivirals, antibacterials, and immunoglobulin with or without corticosteroids. Patients in the hydroxychloroquine group received hydroxychloroquine sulfate $200 \mathrm{mg}$, twice a day for 5 days in addition to the standard treatment. Results indicated that hydroxychloroquine helped reduce the time until body temperature returned to normal, it decreased the duration of cough, and it improved lung imaging findings (16). The above studies together favors the usefulness of hydroxychloroquine in treating COVID-19.

A point worth noting is that a pilot study (17) in China found that hydroxychloroquine might not help promote virus-negative conversion, reduce the time until body temperature returned to normal, or prevent disease progression. The pilot study involved 30 patients who were randomly divided into a hydroxychloroquine group and a control group at a ratio of 1:1. Patients in the control group received antivirals recommended in the Guidance including IFN- $\alpha$ (100\% of patients), arbidol (66.7\% of patients), and lopinavir/ritonavir (13.3\% of patients). Patients in the hydroxychloroquine group received hydroxychloroquine $400 \mathrm{mg}$ once a day for 5 days (100\% of patients), IFN- $\alpha$ (100\% of patients), and arbidol ( $80 \%$ of patients). Results indicated that on day 7 post-inclusion the virus-negative rate was $86.7 \%$ in the hydroxychloroquine group and $93.3 \%$ in the control group (17). The median time until virusnegative conversion, the time until body temperature returned to normal, and the rate of disease progression were comparable in both groups (17). As patients in both groups received multiple antivirals except for hydroxychloroquine and prognosis was good for all patients, distinguishing the effects of hydroxychloroquine from those of the other antivirals used is difficult. Another possibility is that the combination of antivirals had a ceiling effect since both groups had a high rate of virus-negative conversion.

In general, completed clinical studies have yielded relatively promising results regarding the efficacy and safety of chloroquine/hydroxychloroquine in the treatment of COVID-19. More randomized control clinical studies are warranted to verify the value of these two drugs. To obtain clear and strong evidence, the World Health Organization is launching multi-country clinical trials to test the feasibility of chloroquine/ hydroxychloroquine in treating COVID-19, and those results are keenly awaited.

\section{References}

1. COVID-19 Map. https://coronavirus.jhu.edu/map.html (accessed April 6, 2020).

2. National Health Commission. Interpretation of the Sixth Edition of the Guidance for COVID-19: Prevention, Control, Diagnosis, and Management. http://www.nhc. gov.cn/xcs/fkdt/202002/54e1ad5c2aac45c19eb541799bf6 37e9.shtml (accessed April 2, 2020). (in Chinese)

3. Lenzer J. Covid-19: US gives emergency approval to hydroxychloroquine despite lack of evidence. BMJ. 2020; 369:m1335.

4. COVID-19: chloroquine and hydroxychloroquine only to be used in clinical trials or emergency use programmes. European Medicines Agency. https://www.ema.europa. eu/en/documents/press-release/covid-19-chloroquinehydroxychloroquine-only-be-used-clinical-trialsemergency-use-programmes_en.pdf (accessed April 6, 2020).

5. The Multicenter Collaboration Group of Department of Science and Technology of Guangdong Province and Health Commission of Guangdong Province for Chloroquine in the Treatment of Novel Coronavirus Pneumonia. Expert consensus on chloroquine phosphate for the treatment of novel coronavirus pneumonia. Zhonghua Jie He He Hu Xi Za Zhi. 2020; 43:E019. (in Chinese)

6. Gao J, Tian Z, Yang X. Breakthrough: Chloroquine phosphate has shown apparent efficacy in treatment of COVID-19 associated pneumonia in clinical studies. Biosci Trends. 2020; 14:72-73.

7. Notice on Issuance of the Sixth Edition of the Guidance for COVID-19: Prevention, Control, Diagnosis, and Management. National Health Commission. http://www. nhc.gov.cn/yzygj/s7653p/202002/8334a8326dd94d32 9df351d7da8aefc2.shtml (accessed April 2, 2020). (in Chinese) 
8. Notice on Issuance of the Seventh Edition of the Guidance for COVID-19: Prevention, Control, Diagnosis, and Management. National Health Commission. http://www. nhc.gov.cn/yzygj/s7653p/202003/46c9294a7dfe4cef80dc7 f5912eb1989.shtml (Accessed April 2, 2020). (in Chinese)

9. National Health Commission. Audio transcript of a news briefing held by the State Council of China on March 17, 2020. http://www.nhc.gov.cn/xcs/fkdt/202003/01426fc059 0249ecac89a2874214e523.shtml (accessed April 6, 2020). (in Chinese)

10. Huang M, Tang T, Pang P, et al. Treating COVID-19 with Chloroquine. J Mol Cell Biol. 2020.

11. International meeting to share experiences to prevent and control COVID-19. https://e.dxy.cn/broadcast/live/ id/13818? source=pagesidebar (Accessed April 8, 2020).

12. Liu J, Cao R, Xu M, Wang X, Zhang H, Hu H, Li Y, Hu Z, Zhong W, Wang M. Hydroxychloroquine, a less toxic derivative of chloroquine, is effective in inhibiting SARSCoV-2 infection in vitro. Cell Discov. 2020; 6:16.

13. Gautret $\mathrm{P}$, Lagier JC, Parola $\mathrm{P}$, et al. Hydroxychloroquine and azithromycin as a treatment of COVID-19: results of an open-label non-randomized clinical trial. Int J Antimicrob Agents. 2020;105949. DOI: 10.1016/ j.ijantimicag.2020.105949.

14. Gautret P, Lagier JC, Parola P, et al. Clinical and microbiological effect of a combination of hydroxychloroquine and azithromycin in 80 COVID-19 patients with at least a six-day follow up: An observational study. Méditerranée
Infection. 2020. https://www.mediterranee-infection. com/wp-content/uploads/2020/03/COVID-IHU-2-1.pdf (accessed April 6, 2020).

15. Zhou F, Yu T, Du R, et al. Clinical course and risk factors for mortality of adult inpatients with COVID-19 in Wuhan, China: A retrospective cohort study. Lancet. 2020; 395:1054-1062.

16. Chen ZW, Hu JJ, Zhang ZW, Jiang S, Han SM, Yan DD, Zhuang RH, Hu B, Zhang Z. Efficacy of hydroxychloroquine in patients with COVID-19: Results of a randomized clinical trial. medRxiv. 2020; doi: https:// doi.org/10.1101/2020.03.22.20040758.

17. Chen J, Liu DP, Liu L, Xu QN, Xia L, Ling Y, Huang D, Song SL, Zhang DD, Qian ZP, Li T, Shen YZ, Lu HZ. A pilot study of hydroxychloroquine in treatment of patients with common coronavirus disease-19 (COVID-19). Zhe Jiang Da Xue Xue Bao. 2020. (in Chinese).

Received April 6, 2020; Revised April 10, 2020; Accepted April 11, 2020.

\section{*Address correspondence to:}

Jianjun Gao, Department of Pharmacology, School of Pharmacy, Qingdao University, Qingdao, China.

E-mail: gaojj@qdu.edu.cn

Released online in J-STAGE as advance publication April 13, 2020. 